\title{
La genèse du système des cultes reconnus : aux origines de la notion française de reconnaissance
}

\section{Rita Hermon-Belot}

\section{Q OpenEdition \\ 1 Journals}

Édition électronique

URL : http://journals.openedition.org/assr/1106

DOI : 10.4000/assr.1106

ISSN : $1777-5825$

Éditeur

Éditions de l'EHESS

\section{Édition imprimée}

Date de publication : 1 janvier 2005

Pagination : 17-35

ISBN : 2-7132-2043-2

ISSN : 0335-5985

Référence électronique

Rita Hermon-Belot, "La genèse du système des cultes reconnus : aux origines de la notion française de reconnaissance ", Archives de sciences sociales des religions [En ligne], 129 | janvier - mars 2005, mis en ligne le 09 janvier 2008, consulté le 01 mai 2019. URL : http://journals.openedition.org/assr/1106 ; DOI : 10.4000/assr.1106

Ce document a été généré automatiquement le 1 mai 2019.

(c) Archives de sciences sociales des religions 


\title{
La genèse du système des cultes reconnus : aux origines de la notion française de reconnaissance
}

\author{
Rita Hermon-Belot
}

1 Quand la République affirme dans le présent intemporel de la loi qu'elle «ne reconnaît, ne salarie ni ne subventionne aucun culte ${ }^{1}$, elle aurait tout aussi bien pu dire, - et sans doute l'a-t-on très bien entendu ainsi sur le moment -, qu'elle n'en reconnaissait plus aucun. Elle rompait en effet avec ce qui avait été la pratique de l'État depuis un bon siècle, et sa propre pratique depuis déjà près de trente ans, en visant une réalité très spécifique. Le quarante-quatrième et tout dernier article de la loi y revenait encore pour le spécifier, « sont et demeurent abrogées toutes les dispositions relatives à l'organisation publique des cultes antérieurement reconnus par l'État ». Et le législateur avait soin de donner le détail des législations successives qui avaient abouti à façonner le statut de ces cultes reconnus tel qu'en vigueur en $1905^{2}$.

2 Certes, la loi de séparation ne constitue pas toute la laïcité française, mais un volet si déterminant, tant du point de vue du cadre législatif que des représentations de cette laïcité, qu'il paraît indispensable de savoir ce qu'ont été les cultes reconnus pour espérer la comprendre. L'évocation des cultes reconnus nous est familière et peut-être même de plus en plus familière depuis certains débats récents à travers lesquels ils semblent opérer une remontée dans les représentations contemporaines sous les formes diverses de la permanence, de la survivance ou de la résurgence ${ }^{3}$. Mais cette impression de familiarité trouve également à se conforter dans l'image plutôt lisse que renvoie l'historiographie. Les cultes reconnus sont en effet régulièrement présentés comme une pièce de la reconstruction napoléonienne : à la fois, dans la mesure où Bonaparte se pose en héritier de la Révolution, l'entreprise donnant corps à l'héritage révolutionnaire et un ensemble d'une stabilité et d'une longévité rare en France puisqu'il serait resté en vigueur tout au long du xix siècle jusqu'en 1905. Ce qui établit l'empreinte que cette longue étape a forcément laissée dans le patrimoine comme dans la physionomie des 
cultes en France, mais postule aussi une identité entre les cultes reconnus de Bonaparte et ceux que la loi aurait trouvé pour les abolir en 1905.

3 Cette historiographie est plus hésitante en revanche quant aux dénominations sur lesquelles elle s'appuie, puisqu'elle use d'au moins quatre termes en composant des mariages plutôt changeants. Ainsi, penchant volontiers du "régime " au "système ", balance-t-elle entre le singulier du « concordataire » et le pluriel des « reconnus » ${ }^{4}$. Tous termes qui ont en tout cas bien en commun de renvoyer au moment napoléonien et surtout à l'esprit de cohérence et de système propre à toute l'œuvre napoléonienne.

C'est précisément sur l'idée d'un esprit de système du créateur, d'une cohérence pensée d'emblée comme telle, que nous voudrions revenir ici avec une attention d'autant plus vive que ces cultes reconnus semblent constituer une référence plus ou moins explicite dans le débat contemporain. Sans chercher nécessairement à la mettre en doute, nous voudrions tenter d'éclairer les ressorts de cette cohérence en identifiant à la fois les moments de sa construction et les registres dans lesquels elle s'est inscrite, à commencer par l'époque dite fondatrice. Les cultes reconnus que nous connaissons sont-ils ceux de Napoléon, ceux de 1905, ou plutôt ceux qui ont fait tout au long du xix l'objet d'une construction rétrospective dont nous avons gardé le souvenir fixé en 1905 ? Mais aussi, comment évaluer dans la genèse de ce système la part qu'a pu y prendre le principe de reconnaissance et en conséquence la manière dont ce principe a pu s'enraciner dans l'histoire française?

La construction napoléonienne

Dans son tout premier état, ce régime est né de la conviction de Bonaparte, Premier consul des Français, que le rétablissement de la paix et de l'ordre au sortir de la Révolution, et singulièrement l'établissement de son propre pouvoir, passaient nécessairement par la solution des conflits que cette Révolution avait ouverts autour des questions religieuses. On sait qu'en la matière, l'héritage de la Révolution fut aussi complexe que déterminant. L'article $\mathrm{X}$ de la Déclaration des droits proclamait la liberté religieuse et la Constitution de septembre 1791 reconnaissait à chacun «la liberté d'exercer le culte religieux auquel il est attaché " ${ }^{5}$. L'égalité civile était garantie aux protestants en décembre 1789 et aux juifs le 27 septembre $1791^{6}$. Mais les conflits ouverts par les difficultés d'application de la "Constitution civile du clergé ", réforme du statut civil de l'Église votée en juillet 1790, qui constituait en fait une suite de la décision de la Constituante de mettre les biens du clergé à la disposition de la nation pour résoudre la crise financière, ont suscité une crise très grave parce qu'elle s'est étendue à l'ensemble du corps social. Peu à peu, d'alliée de la Révolution qu'elle avait été à de nombreux titres depuis l'été $1789^{7}$, la religion apparut à beaucoup comme suspecte sinon ennemie. L'emballement dramatique de 1793 mena à une vaste révolte contre la religion en général et l'Église catholique en particulier, connue sous le nom de Déchristianisation, mouvement violent mais loin d'avoir été uniforme et universel, et dont il est bien difficile de situer la fin. Après la chute de Robespierre, la Convention thermidorienne décida en septembre 1794 que la République ne salariait plus aucun culte et vota en février 1795 une loi, dite "de la liberté des cultes». Ces dispositions sont souvent considérées comme ayant formé la première séparation de l'Église (exclusivement catholique pour cette fois) et de l'État en France, et elles instaurent en tout cas la première expérience d'une véritable égalité des statuts des différentes religions pratiquées en France. Mais les gouvernements successifs menèrent des politiques religieuses ambiguës, faites d'alternance de mesures d'apaisement et de législations répressives envers les prêtres et 
pasteurs. Au moment où Bonaparte prit le pouvoir, le culte était donc presque partout totalement désorganisé, ses bâtiments fermés ou détournés de leur usage et les pasteurs bien souvent réduits à la misère. Il fut le premier à apporter un soulagement sensible à cette situation en abolissant l'essentiel des législations répressives. Mais les conflits hérités de la Révolution et surtout les divisions à la fois religieuses et politiques au sein d'une catholicité française bouleversée ne s'en firent que plus durement sentir, menant Bonaparte à la certitude que seul le Pape pouvait obtenir la réconciliation des Français. $C^{\prime}$ 'est à cet effet que furent ouvertes des négociations avec Rome, renouant une relation que la papauté avait considérée comme rompue par l'adoption de la Constitution civile du clergé. Négociations qui menèrent à la signature de la Convention passée à Paris le 26 messidor an IX, 15 juillet 1801, entre « sa sainteté Pie VII et le gouvernement français » et connue sous le terme de Concordat ${ }^{8}$.

6 Affirmant que la religion catholique, apostolique et romaine était «la religion de la grande majorité des citoyens français », le Gouvernement de la République garantissait qu'elle serait «librement exercée en France» et que son culte serait "public, en se conformant aux règlements de police». Dix-sept articles s'appliquaient à régler les relations entre l'État et l'Église catholique.

7 Ce texte ne constituait toutefois qu'un cadre général bien loin de pouvoir résoudre tous les problèmes en suspens et sa mise en application se trouva considérablement retardée par les lenteurs de la Curie romaine, mais aussi par l'hostilité d'une classe politique dont beaucoup de membres s'étaient crus définitivement débarrassés de tout lien entre religion et État et partageaient avec les milieux protestants la crainte de voir la religion catholique retrouver une position dominante. C'est à la faveur de ce délai imprévu et plusieurs fois prolongé, et au prix de multiples transactions, que devaient surgir les «Articles organiques». Soixante-dix-sept articles concernant le culte catholique et quarante-quatre concernant les deux cultes protestants mettaient la même minutie à régler la vie des institutions religieuses et celle des fidèles. Le conseiller d'État « chargé de toutes les affaires concernant les cultes» nommé en octobre 1801, Jean-Étienne Portalis, les présenta aux Assemblées consulaires en avril 1802, pour être votés avec le Concordat comme un seul et même "projet de loi » de la République. Le Saint-Siège n'avait guère été consulté.

8 Les Articles organiques donnent une inscription institutionnelle effective au pluralisme, mais il s'agit là d'un pluralisme exclusivement chrétien. Portalis le dit très clairement en présentant l'ensemble Concordat-Articles organiques au Conseil d'État, le gouvernement avait écarté l'idée de légiférer pour le culte juif, admis au bénéfice de la liberté des cultes sans aucune attention ou intervention particulière de l'État ${ }^{9}$. La décision de contrevenir à une telle détermination fut le fruit de préoccupations et requêtes d'ordre très divers. Les premières émanèrent des juifs eux-mêmes.

Alors que l'application de la loi de 1791 supposait la dissolution des institutions communautaires traditionnelles, la Déchristianisation avait désorganisé ce culte comme les autres. Les anciens dirigeants des communautés s'adressèrent aux autorités locales et nationales, les suppliant d'organiser le culte juif comme elles l'avaient fait pour les cultes chrétiens. Mais ces demandes éveillèrent une vive campagne, initiée par un des plus éloquents hérauts de la Contre-Révolution, le comte Louis de Bonald, qui dressait le terrible tableau d'une Alsace saignée à blanc par l'« usure juive», en stigmatisant la loi qui avait accordé la citoyenneté aux juifs comme une des pires "erreurs» de la Révolution. Un certain nombre de ces ouvrages parvinrent directement à l'Empereur ${ }^{10}$ et 
c'est dans une perspective de prophylaxie sociale que celui-ci prit la décision de légiférer. Il s'agissait de disposer de lois d'exception pour soustraire les régions de l'Est à l'emprise juive. Seule la résistance de certains des membres de son Conseil d'État put engager l'Empereur à considérer également les demandes des juifs et même à prendre la décision très soudaine de réunir à cet effet une «synagogue juive » à Paris. Il y eut en fait deux assemblées : l'Assemblée des notables, qui affirma la loyauté des juifs de France à l'État et établit le plan d'organisation du culte, et le fameux "Grand Sanhédrin » napoléonien chargé d'apporter une caution religieuse dont Napoléon s'imaginait qu'elle aurait valeur d'obligation pour tous les juifs de l'Empire. L'élaboration des décrets fut ensuite renvoyée au Conseil d'état qui s'en acquitta en mars 1808. Les deux premiers fixaient l'organisation du culte juif en créant le système des consistoires. Un troisième enserrait la vie économique des juifs dans un dur réseau de contraintes spécifiques pour une période probatoire de dix ans ${ }^{11}$.

La religion juive faisait effectivement l'objet d'une législation nationale prévoyant l'organisation du culte, mais celle-ci n'avait été que le corollaire inévitable d'un projet législatif qui était d'abord et avant tout un projet de dure police. C'est d'ailleurs sous les auspices non du ministre des cultes mais du ministre de l'Intérieur que s'est effectué tout le travail d'élaboration de ces mesures.

Quelles qu'aient pu être les intentions qui y ont présidé et au-delà même du sort des principaux intéressés, l'extension de l'action législative représente une étape inédite et décisive dans la mesure où l'État acceptait de se donner un interlocuteur religieux qui ne soit pas chrétien. La régulation du culte juif passait sous le contrôle du ministère des cultes, la mention « non catholiques » englobant cette fois des cultes chrétiens et un culte non-chrétien.

Un certain nombre de prescriptions étaient adressées de façon identique au culte catholique, aux deux cultes protestants et au culte juif (qui prenait le nom d'« israélite »), à commencer par la désignation qui leur devenait commune à tous de «ministres du culte" pour désigner leurs officiants, même si elle englobait des pratiques fort différentes. Les bonnes relations entre les cultes n'étaient pas seulement envisagées dans une sphère publique neutralisée, les cultes en tant que tels faisaient leur entrée de fait dans un monde commun. Ainsi, les Articles organiques prévoyaient-ils que les curés « ne se permettront dans leurs instructions, aucune inculpation directe ou indirecte, soit contre les personnes, soit contre les autres cultes autorisés dans l'État ${ }^{12}$. Mais une telle disposition fait entendre aussi la manière dont le pouvoir entendait désigner ces cultes. Les Articles organiques de même que le Code pénal de $1811^{13}$ évoquaient essentiellement des «cultes autorisés». Alors que, dans son discours de présentation de 1802, Portalis passait d'une "surveillance » à la fois "utile » et "protectrice », à la "protection éclairée » dont feraient l'objet des cultes " protégés ».

13 Protection qui, à des degrés et selon des modalités diverses, comportait pour tous une même autorité intrusive de l'État, une même immixtion, particulièrement la même pression pour l'unification des pratiques, des rituels et des enseignements (un seul catéchisme pour tous les catholiques), une véritable uniformisation interne et la délégation obligée d'une fonction de police interne. Le principe de la législation supposait enfin, et celle-ci spécifiait même parfois très précisément, qu'aucun culte ne pourrait procéder au moindre changement sans l'accord de l'État. donner une image commune, c'est-à-dire surtout de mettre en image la bienveillance 
pluraliste de l'Empereur et la gratitude des fidèles. Une telle gratitude ne doit cependant pas être réduite à une mise en scène du pouvoir. Il faut compter bien sûr avec le joug de fer du gouvernement impérial. Mais, sans vouloir minimiser aucunement toutes les réclamations et protestations qu'ont pu susciter nombre de dispositions des nouvelles législations, à commencer par la publication unilatérale des Organiques par le gouvernement français, le mouvement qui l'emportait semble bien avoir été l'assentiment, avec une adhésion encore plus enthousiaste de la part des membres des cultes minoritaires ou du moins de ceux qui parlaient en leur nom. Une adhésion dont on peut d'ailleurs d'autant mieux mesurer l'élan à l'immense effort d'adaptation et aux nombreux sacrifices qu'elle comportait.

Dans quelle mesure pour autant l'ensemble atteignait-il un niveau de cohérence qui puisse en faire une manière de « système " ?

Les statuts des cultes étaient très divers. Une diversité bien plus grande d'ailleurs que ne l'aurait voulu un gouvernement qui avait notamment pu envisager un statut unique pour les deux cultes protestants, mais bien nécessaire pour répondre un tant soit peu à la nature et aux besoins spécifiques de ces cultes. Mais une diversité aussi dans les conditions d'existence et d'exercice du culte, qui tenait à une inégalité de traitement que les législations prononçaient très clairement. Ainsi, si tous les cultes avaient bien des " ministres ", la rémunération de ces ministres, rémunération qui non seulement assure d'abord leur existence, mais matérialise aussi leur relation avec l'État et justifie le contrôle exercé par ce dernier, était d'emblée assurée par les Articles organiques pour le culte catholique et les deux cultes protestants, mais elle avait été très expressément refusée au culte juif. Sans revenir non plus aux intentions qui avaient présidé à la décision de légiférer pour ce dernier culte, la forme même de la législation était bien différente entre Articles "organiques" et série de décrets juxtaposant dispositions concernant les cultes et dispositions civiles restrictives à l'encontre d'une catégorie de citoyens.

Le principe d'exception admis par le décret de 1808 a encore eu d'autres effets. À la suite d'une initiative prise en 1809 par la cour de Colmar, s'est généralisée l'exigence d'un serment particulier, dit more judaico, supposant qu'un témoignage apporté par un citoyen de confession israélite ne pouvait avoir valeur juridique sans un appel solennel à la malédiction divine sur le parjure en puissance.

Des disparités que les textes législatifs avaient pourtant évitées ont pu aussi se faire rapidement sentir dans leur interprétation et application, et introduire un traitement différent entre cultes chrétiens. L'affirmation de la suprématie au moins sociale de la religion catholique, "Religion de la grande majorité des citoyens français », n'a pas été sans conséquences sur la mise en œuvre de la première législation consulaire. Ainsi l'article XLV des Articles organiques concernant le culte catholique prohibait-il la tenue de toute cérémonie religieuse hors des édifices consacrés au culte catholique «dans les villes où il $\mathrm{y}$ a des temples destinés à différents cultes ». Mais la portée en fut très vite fortement restreinte, ceci probablement sur l'intervention personnelle de Bonaparte ${ }^{14}$. Une circulaire du ministère de l'Intérieur, en date du 20 avril 1803, en limita l'application aux chefs-lieux d'Église consistoriale. Alors que dans les plus grandes villes, à commencer par Lyon en juillet 1803, le siège du consistoire était placé par fiction juridique dans un faubourg ou une commune de banlieue ${ }^{15}$. Les protestants, puisqu'à cette date ils étaient les seuls non-catholiques à pouvoir invoquer le bénéfice des Organiques, cherchèrent sans succès à obtenir un règlement de police précis. Mais il semble aussi que compte tenu 
du rapport démographique, les responsables des cultes minoritaires aient longtemps jugé plus sage de se résigner, les protestations, cette fois bien plus radicales, ne reprenant qu'à partir des années $1880^{16}$.

La mise en « système » du régime des cultes reconnus

19 La cohérence que nous connaissons aurait donc pris corps bien au-delà de l'Empire. Statuts et conditions d'exercice du culte ont en effet continué à se modifier peu à peu dans le sens d'une égalisation lente, mais continue. Cette évolution se produisit au sein d'un cadre lui-même soumis à des révisions radicales dues aux changements de gouvernement et même de régimes politiques, mais aussi à la tension constante entre une liberté des cultes jamais officiellement remise en cause et la désignation de la religion catholique au moins comme celle de « la majorité des Français ».

C'est très certainement pendant la Restauration que cette dernière tension s'est fait le plus vivement ressentir ${ }^{17}$. La Charte constitutionnelle du 4 juin 1814 portait en effet que " chacun professe sa religion avec une égale liberté et obtient pour son culte la même protection " ${ }^{18}$. Mais elle spécifiait aussi dans l'article suivant que « cependant la religion catholique, apostolique et romaine est la religion de l'État ». L'aporie a été fréquemment soulignée. Elle mériterait même d'être quelque peu éclairée, et elle peut l'être sous un jour tout à fait édifiant à la lumière de ce dialogue entre Talleyrand et sa grande amie Madame de Duras en 1817 :

21 «Savez-vous », demande-t-il, « qui a donné l'avis d'émettre ces articles ? - Non, mais ils sont excellents. - Eh bien, c'est moi. - Je suis enchantée que vous ayez fait cette bonne œuvre et je vous en félicite. - Mais savez-vous pourquoi j'ai donné ce conseil ? - Non, mais je suis certaine que vous aviez une bonne raison. - Eh bien, j'ai suggéré ces mots parce qu'ils ne signifiaient rien du tout. » ${ }^{19}$

Peut-être ne faut-il pas se laisser tout à fait prendre par le sens de la dérision d'un Talleyrand toujours présenté comme l'incarnation même de l'opportunisme et de la versatilité, mais dont l'attitude en ce qui concerne la question de la liberté des cultes a justement été faite de la plus exemplaire constance depuis les premiers temps de la Révolution. Une constance d'autant plus exemplaire qu'il lui aura fallu presque toujours s'inscrire à rebours des mouvements dominants ${ }^{20}$. Aussi contradictoire qu'elle puisse nous paraître, la double affirmation de la Charte aurait donc été largement délibérée et la tension entre héritage révolutionnaire et exigences de la restauration monarchique tout aussi bien assumée, au moins dans l'esprit des acteurs du temps les plus avertis. Elle n'a d'ailleurs pas manqué de produire les effets les plus sensibles.

Chemine en effet ici un processus d'égalisation au sein duquel il nous semble pouvoir discerner deux voies distinctes. La première a opéré par la mise à niveau de ce qui est spécifique : les dispositions concernant les juifs, lesquelles font peu à peu l'objet d'une sorte de "rattrapage». Ce dernier concernait à la fois, mais non de façon continue, l'égalité civile des fidèles des différents cultes et l'égalité des cultes entre eux. Lorsque le décret «infâme " vint à expiration en mars 1818, le gouvernement fit savoir qu'il ne voyait aucun intérêt à sa reconduction et la décision fut entérinée sans difficulté majeure par la Chambre. C'est en 1846, et au terme d'une dure campagne, que fut aboli le serment More Judaico. Restait, malgré l'appui de députés aussi influents que Benjamin Constant, la question des dettes des communautés de l'Ancien régime et particulièrement celle, très lourde, des communautés de l'Est ${ }^{21}$. Elle ne devait jamais être réglée, et les remboursements s'effectuèrent jusqu'à l'annexion de 1870 . 

des rabbins au budget du culte. Et sur ce point déterminant, la différence est nette entre Restauration et Monarchie de juillet. La Charte constitutionnelle de 1814 prorogeait les dispositions impériales : « Les ministres de la religion catholique, apostolique et romaine, et ceux des autres cultes chrétiens, reçoivent seuls des traitements du Trésor royal ${ }^{22}$. Alors que la Charte de 1830, reprenant une formulation très proche, avait soin d'en retrancher l'adjectif « seul ${ }^{23}$. Elle entendait ainsi, expliquera quelques mois plus tard le ministre en charge de l'Instruction publique et des cultes, laisser «à la sagesse de l'autorité civile le droit d'admettre plus tard à ce privilège tels ou tels cultes qui n'auraient pas pu le réclamer sous la Charte ancienne ${ }^{24}$ et la loi est effectivement promulguée le 8 février 1831. À partir de 1839, le culte israélite disposa également d'aumôniers dans les hôpitaux militaires. Enfin, l'ordonnance d'organisation du culte israélite en date du 28 mai 1844, récapitule et ordonne l'ensemble des mesures dont le système consistorial a pu faire l'objet depuis 1808, et elle prévoit pour les juifs la possibilité de recours à la procédure «comme d'abus » ouverte aux catholiques et aux protestants par les Organiques de 1802. Tandis que la qualification d'« organiques » pour l'ensemble de ces dispositions évoque une volonté tardive d'harmonisation.

clutôt dans une autre voie d'evolution du statut des cultes en France que l'on discernera les prémisses de ce qui pourrait faire un véritable esprit de système, dans la mesure où cette évolution cesse de tenir en mesures ad hoc adoptées successivement au bénéfice de tel ou tel culte spécifique, pour prendre la forme d'une extension aux cultes socialement minoritaires de mesures prévues au premier chef pour la religion catholique majoritaire. Et cette extension paraît se faire de plus en plus naturelle et incontournable, après s'être imposée précisément au moment où la religion catholique avait retrouvé le statut de religion de l'État. délits commis par la voie de la presse ou par tout autre moyen de publication », punissait d'une peine de prison ou d'amende quiconque aura outragé ou tourné en dérision la religion de l'État. Mais la suite de l'article prévoyait les mêmes peines « contre quiconque aurait outragé ou tourné en dérision tout autre religion dont l'établissement est légalement reconnu en France». Et l'extension était d'ores et déjà prévue dans la première présentation du projet à la Chambre des députés par le Garde des Sceaux, M. de Serre, le lundi 3 décembre $1821^{25}$. Au moins dans l'esprit de certains des auteurs du projet, l'intention pluraliste était bien claire, l'article avait pour but de punir des cris, tels que ceux-ci : «à bas les prêtres ! à bas les nobles ! à bas les boulangers ! à bas les juifs ! à bas les protestants! à bas les catholiques! $»^{26}$.

Même la terrible loi dite « du sacrilège » du 20 avril 1825, instaurant « la répression des crimes et des délits commis dans les édifices ou sur les objets consacrés à la religion publique ", aboutit paradoxalement à étendre, non bien évidemment la notion de sacrilège, mais les dispositions prévues à l'encontre du vol sacrilège et des délits commis dans les églises ou sur les objets consacrés à la religion à tous les cultes « légalement établis en France ${ }^{27}$. De même, les possibilités d'exemptions et remplacements prévues par les lois du 22 mars 1831 sur le service dans la Garde nationale et du 21 mars 1832, "sur le recrutement de l'armée ", s'offraient d'abord aux élèves des grands séminaires, mais aussi aux «jeunes gens autorisés à continuer leurs études pour se vouer au ministère dans les autres cultes salariés par l'État ${ }^{28}$. Dernière formulation bien significative d'ailleurs du soulagement que le Législateur a trouvé, une fois adopté le 
principe du traitement des rabbins, à pouvoir s'appuyer sur une catégorie des cultes homogène et définie par un critère objectif.

Il semble bien que, de loi en loi, se fasse jour une continuité que la succession accélérée des régimes politiques dans la France de la première moitié du xixe siècle n'a jamais pu interrompre. Et il semble surtout, qu'à partir de l'équilibre si délibérément paradoxal institué sous l'inspiration de Talleyrand, les exigences de la religion d'État aient abouti à entraîner le statut des autres cultes. La Restauration aurait ainsi vu exigence d'une religion de l'État et exigence pluraliste se redoubler l'une l'autre, pour fusionner une partie au moins de leurs réquisits, en une synergie inattendue mais efficace.

Une autre étape pourrait encore avoir été atteinte dans l'évolution de la manière de concevoir ce que pourrait devenir l'extension. On a dit comment la Charte de 1830 envisageait le principe de l'ouverture de ce qu'elle appelait encore un "privilège », mais elle le faisait pour un culte déjà bien connu. En affirmant que «les ministres, soit des cultes actuellement reconnus par la loi, soit de ceux qui seraient reconnus à l'avenir, ont le droit de recevoir un traitement de l'État ", c'est un avenir libre de toute détermination et par nature inconnu, que la constitution de 1848 ouvrait à son tour ${ }^{29}$. À partir du moment où était envisagée l'ouverture à d'autres cultes qui seront alors admis à bénéficier d'un statut déjà défini préalablement à toute demande circonstanciée, ce régime des cultes atteindrait une cohérence interne qui en ferait un dispositif abstrait et intangible. Mais la constitution de 1848 a été bien vite abrogée et les virtualités du système guère mises en œuvre jusqu'à nos jours dans les régions restées " concordataires ${ }^{30}$. Il nous faut donc revenir à l'usage que l'on prétendait faire d'un tel système et surtout aux avantages que l'on en attendait.

De fait, ce n'est qu'une fois clarifiée la question des traitements que son importance peut se dire clairement, tandis que le débat, d'autant plus significatif qu'il se jouait essentiellement entre orateurs tous d'accord entre eux pour le vote de la loi, esquissait une véritable doctrine des cultes reconnus. Ce qui confirme à la fois la puissance dynamique que la présence juive a pu receler dans le contexte français mais aussi à quel point les débats ont dépassé le sort des seuls intéressés, pris plus ici en tant que minorité non chrétienne que pour eux-mêmes. La discussion sur l'accès des rabbins à un « salaire public » posait notamment la question des critères, de « règles » que le ministre Mérilhou aurait aimé pouvoir " préciser d'une manière invariable ». Certes, celles qu'il proposait pour sa part s'appliquaient particulièrement bien au cas israélite parce qu'il ne voulait se placer que du point de vue des besoins et des mérites des éventuels candidats ${ }^{31}$. Mais le dernier mot devait revenir à Joseph-Marie Portalis dit «le jeune», le fils du précédent, qui prit la parole au nom de la commission chargée d'examiner le projet de loi en adoptant une perspective largement décalée par rapport à celle du ministre. Car aux yeux de Portalis, « le salaire public des ministres d'un culte est accordé dans l'intérêt de l'État plus encore que dans l'intérêt du culte même ${ }^{32}$.

31 Ces propos devaient effectivement s'avérer définitifs. Deux décennies plus tard, les années 1850 ont été à la fois un moment de révision et de codification des statuts des cultes minoritaires ${ }^{33}$ et surtout, elles ont été un grand moment de mise en forme de la doctrine. Et c'est bien à Portalis que revenait le rédacteur du grand répertoire Dalloz, bible de la jurisprudence, particulièrement lorsqu'il tenait à remarquer que les traitements des ministres des cultes «ont pour objet, en maintenant les institutions religieuses, en assurant le service public des cultes, en accordant à ceux de la grande majorité des Français l'appui et le secours que réclame leur importance, de mettre l'État 
mieux à portée d'exercer le droit de surveillance qui lui appartient sur les matières religieuses et la conduite des ministres des cultes ${ }^{34}$. On ne saurait rêver plus forte et claire définition de ce que Dalloz appelle à longueur de page le «système » des cultes reconnus ou d'ailleurs "système du Concordat», et qu'il n'a de cesse, en en faisant l'apologie, de renvoyer à la continuité avec l'œuvre napoléonienne. Ce dont on ne saurait s'étonner à l'aube du Second Empire.

La reconnaissance des Reconnus

Quelle place a pu revenir au sein d'une telle construction à la notion de reconnaissance et surtout quel champ celle-ci a-t-elle pu y recouvrir?

La Révolution avait également ouvert cette question-là. En plaçant toute réflexion politique dans la perspective des droits, elle introduisait à la fois le lexique et la démarche de la reconnaissance. On connaît la formule qui clôt le préambule de la Déclaration des droits de l'homme et du citoyen pour annoncer l'énumération de ces droits: «En conséquence, l'Assemblée nationale reconnaît et déclare, en présence et sous les auspices de l'Être suprême, les droits suivants de l'Homme et du Citoyen ».

La question de la reconnaissance s'est bien vite posée à propos de la religion et particulièrement celle de la reconnaissance du religieux par le politique. Il s'agissait notamment de savoir si, après avoir mis les biens du clergé à la disposition de la nation, l'Assemblée constituante se devait de déclarer officiellement la religion catholique religion nationale du royaume. Elle a à la fois évité de le faire et évité de s'y refuser à haute voix, en considérant cette reconnaissance comme implicite dans la mesure où les prêtres catholiques étaient rémunérés par l'état. De fait, les partisans les plus intransigeants d'une religion nationale voulaient plutôt la faire "décréter " ou "déclarer ", alors que le lexique de la reconnaissance semblait pencher de plus en plus nettement du côté du politique. Et il y penchait encore davantage lors de ce moment de 1795 si souvent vu comme une première séparation. Le rapporteur de la loi, Boissy d'Anglas ${ }^{35}$, préconisait de « reconnaitre " les droits des citoyens alors que sa position à l'égard de la religion semblait bien faire jouer un principe de non-reconnaissance et même de non-connaissance. "En ne considérant la religion que comme une opinion privée ", expliquait-il, "vous ignorerez ses dogmes, vous regarderez en pitié ses erreurs, mais vous laisserez à chaque citoyen la faculté de se livrer à son gré aux pratiques de celle qu'il aura choisie ». Le texte voté spécifiait effectivement que « la loi ne reconnaît aucun ministre du culte». Le politique se réservait pour sa part d'exiger la plus expresse reconnaissance de la part du religieux lorsqu'il prescrivait le serment suivant: "Je reconnais que l'universalité des citoyens français est le souverain, et je promets soumission et obéissance aux lois de la République ${ }^{36}$.

En revanche, en tout cas dans sa première étape, le dispositif fondateur mis en place par l'administration napoléonienne s'appuyait sur une procédure de reconnaissance. C'est sur un échange de reconnaissance réciproque que s'ouvrait la Convention entre le Pape et le Gouvernement français. D'une part, « le Gouvernement de la République reconnaît que la religion catholique, apostolique et romaine, est la religion de la grande majorité des citoyens français ». Et de l'autre, "Sa Sainteté reconnaît également que cette même religion a retiré et attend encore en ce moment le plus grand bien et le plus grand éclat de l'établissement du culte catholique en France et de la profession particulière qu'en font les Consuls de la République ». Et les dix-sept articles du Concordat étaient présentés comme fondés, en conséquence, sur «cette reconnaissance mutuelle». Cet échange 
s'inscrit également dans le cadre de relations d'État à État. C'est sous l'égide du ministère des Affaires étrangères que la négociation a été menée et le texte signé.

Reste que par la suite rien dans les Articles organiques considérés comme la véritable armature des cultes reconnus ne revenait à cette notion de reconnaissance, et bien peu de choses d'ailleurs dans le discours du temps. Dans un « rapport» de l'an XI sur le régime des monitoires, Portalis expliquait bien que ce «moyen» ne faisait que dormir «tant qu'on ne reconnaissait aucun culte » et qu'« il renait avec les idées religieuses ». Mais ces quelques évocations restent bien fugitives et isolées ${ }^{37}$. L'occurrence la plus significative revenant à Napoléon lui-même, lorsqu'il évoquait devant son Conseil d'État « les religions catholique, réformée et luthérienne reconnues par le Concordat ${ }^{38}$. La vision était très généreusement extensive car si le Concordat acceptait de limiter la reconnaissance de la religion catholique à celle de la très grande majorité des Français, ceci ne revenait pas tout à fait à reconnaître positivement celle des réformés et luthériens. Napoléon faisait d'ailleurs non pas de l'État français mais du Concordat l'acteur de cette reconnaissance. $C^{\prime}$ 'est effectivement lui qui a mis en scène l'équivoque sur laquelle reposait ce régime dit " concordataire ", et il l'a fait très sciemment. La pointe du discours impérial était de plus toute restrictive car Napoléon entendait surtout dire ici ce dont il ne voulait pas : d'une part d'une religion dominante, mais surtout "qu'il s'en établisse de nouvelles », ceci devant un Conseil précisément réuni pour examiner les solutions à apporter à ce qui était considéré comme le problème posé par la présence juive.

La plupart des historiens considèrent cependant que « la religion juive a été reconnue par les 3 décrets du 17 mars 1808 ", voyant sans doute une reconnaissance de fait dans la mesure où il a été légiféré pour elle ${ }^{39}$. Ils suivent d'ailleurs en cela la pensée des auteurs de doctrine, mais ces derniers n'ont pas fait mystère du souci qui les animait de renvoyer le «système » dont ils se faisaient si ardemment les apologètes à l'illustre fondation napoléonienne ${ }^{40}$.

38 L'Empereur avait pourtant été extrêmement clair dans son refus d'une dimension essentielle de la reconnaissance, cette dimension «symbolique » à laquelle on sait par ailleurs combien il a pu être attentif. Ainsi, si les membres des cours d'appel assistaient aux processions de la Fête Dieu et si le sacre mobilisa la pompe catholique, on veilla avec le plus grand soin à y inviter des représentants des cultes protestants. Les responsables des organisations juives avaient, pour leur part, parfaitement compris le sens d'une aussi visible association à l'appareil d'État. Mais lorsque les consistoires israélites nouvellement créés se crurent autorisés à arborer l'emblème impérial dans leur correspondance et sur leurs actes, l'initiative fut très mal reçue et un tel usage leur fut très vertement interdit le 26 juin 1809. L'Empereur ne devait d'ailleurs accorder qu'une seule audience aux représentants des juifs, pour s'y montrer fort brutal et s'opposer catégoriquement à ce qu'il en soit fait mention publique. En revanche, le roi de la Restauration, Louis XVIII, saisissait dès 1816 l'occasion de la nouvelle année pour accorder aux représentants des consistoires la reconnaissance publique d'une réception officielle à la cour.

C'est bien à travers le processus d'extension des " privilèges » accordés aux cultes que le lexique et l'idée de la reconnaissance devaient eux aussi faire leur chemin. Non d'ailleurs sans adversité, car chaque tournant eut son lot de tentatives pour faire coïncider reconnaissance sociale et reconnaissance de vérité ${ }^{41}$. Mais même au prix des plus âpres débats, la dissociation des deux finit invariablement par l'emporter. «reconnu» semblant d'abord s'être plus volontiers appliqué à l'établissement. 
L'évolution concerne alors la réalité que désigne ce terme d'« établissement ». Elle prit peu à peu un sens de plus en plus étendu, du simple édifice en l'An $\mathrm{XI}^{42}$, aux établissements ecclésiastiques désignés par la loi du 2 janvier 1817 sur les donations et legs à « tout établissement ecclésiastique reconnu par la loi », et à l'extension de la loi de 1822 sur la presse à « tout autre religion dont l'établissement est légalement reconnu en France ». Ce qui n'empêcha nullement la coexistence prolongée du terme de "reconnu » avec celui d'« établi », notamment sous la plume de Portalis jeune, déjà rapporteur de la Commission chargée de l'examen du projet en février 1822.

41 Mais justement, si Joseph-Marie Portalis a tenu dans le débat de 1831 à reprendre un à un les propos de son ministre, c'est aussi et peut-être surtout afin d'y donner une véritable théorie de la reconnaissance. Le "privilège » que constitue le traitement par l'État va en effet selon lui bien au-delà de la protection chère à son propre père. C'est même tout autre chose que «le signe de respect de l'autorité civile pour toutes les croyances religieuses» que le ministre venait d'évoquer. Aux yeux de Portalis, c'est «une reconnaissance publique de la secte qui l'obtient, c'est un établissement qu'on lui accorde, ce sont des lettres de grande naturalisation qu'on lui donne; c'est une homologation solennelle de sa doctrine et de ses dogmes, dont on encourage la propagation et dont on assure l'enseignement ${ }^{43}$.

On trouvera certes une dimension symbolique à voir le fils achever ainsi l'œuvre du père, mais en remarquant précisément son insistance à apporter à l'édifice un pan tout à fait neuf. Ceci, alors que ce fils avait grand soin de rappeler qu'il avait déjà été très activement engagé dans cette carrière par son père et savait donc très bien ce que ce père avait voulu faire ou ne pas faire ${ }^{44}$.

43 Dans la mesure où il semble que la loi de 1822 ait ouvert pour la première fois ce terme de « reconnus », la dynamique enclenchée par les besoins de la religion d'État se fit sentir aussi dans la démarche de reconnaissance. Mais cette reconnaissance ne prit son sens spécifique, elle ne fut définie en elle-même qu'au moment où le régime prit effectivement corps et où s'opéra sa cristallisation en système cohérent offrant à ses bénéficiaires des conditions unifiées; ceci tout au moins au plan institutionnel accessible à la loi.

Mais on remarquera également que l'émergence et la naturalisation de ce qualificatif de « reconnus » se sont faites en 1822 dans le contexte des « religions » et non des «cultes». Alors que Joseph-Marie Portalis, évoquant en 1831, la «secte», sa "doctrine» et ses « dogmes » était bien loin lui aussi de s'en tenir à une simple reconnaissance sociologique. Et si le rédacteur du Dalloz traite bien pour sa part en 1853 de «cultes » reconnus, il n'hésite cependant pas à affirmer qu'« il y a un milieu entre la foi et l'indifférence pour l'État », et que " c'est ce milieu qui sert de base au système de la protection " ${ }^{45}$. Le terme de « reconnus » revêt un sens qui dépasse celui de la simple reconnaissance légale. Mais l'affirmation du principe de reconnaissance dit, en même temps que l'ouverture, les délimitations du système.

Le processus d'accréditation de cette épithète de «reconnus» a d'ailleurs été très efficacement renforcé sur un plan où l'institutionnel et le symbolique se rencontrent presque exactement, celui d'une définition négative alimentée par la loi et surtout par l'abondante jurisprudence à laquelle son application a donné lieu. Car les activités des cultes qui n'ont pas eu accès au statut d'«autorisés » sont bien souvent stigmatisées comme tombant sous le coup de la législation à l'encontre des associations illicites ${ }^{46}$. Dans le principal reproche que leur adresse le rédacteur du Dalloz, le fait de constituer des «nouveautés religieuses dont le nom est inconnu à la loi », apparaît une exigence de 
l'État effectivement présente depuis l'époque napoléonienne, celle de la connaissance ${ }^{47}$. Mais, dans une perspective où ce qui est connu de l'État et même reconnu par lui ne peut plus être modifié sans son accord.

Le régime des cultes reconnus s'avère bien rétif à toute tentative d'évaluation univoque. Alors que la délégation de pouvoir confiée par l'État aux cultes reconnus assure le plus efficace contrôle interne de toute lecture éventuellement dissidente, l'angle obscur du système réside dans l'indétermination des conditions d'exercice qu'il offre aux cultes n'entrant pas dans la définition des Reconnus. En cela, et jusque dans sa consécration la plus tardive, le système répondait parfaitement au dessein de Bonaparte. La religion y constituait un pilier du gouvernement et de l'ordre social que ce dernier entendait garantir. Et si, au sein du cadre ainsi tracé, la volonté de débordement, et notamment les tentatives pour faire imposer la loi d'une écrasante majorité sociologique, restaient toujours possibles, le système n'avait de sens que dans la mesure où il servait d'abord le pouvoir de l'État.

Mais le projet napoléonien a beau avoir constitué une étape décisive de la constitution du système des cultes reconnus, il ne saurait pour autant le résumer à lui seul. Certes, la Révolution qui a été la première à proclamer la liberté des cultes en France n'a pas su faire entrer cette liberté dans les faits et, dans sa véritable version libérale, celle de 1789-1791, l'héritage révolutionnaire a été bien long à mettre en œuvre. Mais du moins, une fois affirmé le principe de la liberté des cultes en France, celui-ci n'a-t-il plus jamais été remis en cause. L'étape napoléonienne montre peut-être d'ailleurs surtout la capacité de résistance de ce principe, même à un Napoléon dont l'image d'héritier de la Révolution, défenseur de la liberté des cultes, doit beaucoup à une propagande qui a su ériger en projet cohérent une suite de mesures largement dépendantes des circonstances. Reste, qu'en vertu de la volonté de l'Empereur ou contre elle, et en dépit de ses restrictions, le régime napoléonien finit par se rapprocher de fait d'une égalité de statuts entre cultes chrétiens et non chrétiens dont il faut rappeler combien elle restait exceptionnelle dans l'Europe du temps ${ }^{48}$. Enfin et surtout, le régime né des législations napoléoniennes n'eut de cesse d'assurer son propre renouvellement pour n'atteindre une véritable stabilisation qu'au moment où il parvenait à donner sa plus parfaite expression à cette égalité.

Car la trajectoire des cultes reconnus est également le témoin de l'action d'une tendance libérale à l'œuvre de façon quasi continue depuis la Révolution jusqu'au sein des régimes politiques qui ont voulu afficher le plus hautement leur distance avec l'héritage révolutionnaire. Mais lorsque ce régime des cultes a atteint une véritable cohérence, au moment où s'est définitivement affirmée une réelle égalité entre les cultes concernés, l'idée de reconnaissance prenait également un sens subjectif et symbolique qui dépassait de très loin la simple reconnaissance légale.

\section{NOTES}

1. Loi du 9 décembre 1905 sur la séparation des Églises et de l'État, article 2. 
2. Sur la séparation, voir avant tout Jean-Marie Mayeur, La séparation des Églises et de l'État, Paris, Les Éditions ouvrières, 1991.

3. Ce régime n'a jamais été aboli dans les évêchés de Metz et Strasbourg. Jean Baubérot voit dans certaines dispositions de la loi de 1905 les conditions d'une survivance partielle ; cf. Jean Baubérot, « La laïcité », in Vincent Duclert et Christophe Prochasson, dirs., Dictionnaire critique de la République, Paris, Flammarion 2002, p. 202-208. Survivance encore renforcée par nombre de dispositions législatives récentes qui suggèrent à JeanPaul Willaime l'expression de « régime implicite des cultes reconnus », reprise notamment par Danièle Hervieu-Léger, La religion en miettes ou la question des sectes, Paris, Calmann Lévy, 2001, p. 23 (cf. Arch. 118 - p. 29-44). Enfin, l'éventualité d'une résurgence a été évoquée à maintes reprises lors des débats suscités par la création du Conseil français du culte musulman. Elle a été affirmée de la façon la plus catégorique par Esther Benbassa, La République face à ses minorités, Les Juifs hier, les Musulmans aujourd'hui, Paris, Mille et une nuits, 2004. Cf. notamment le chapitre : " Napoléon version Sarkozy ».

4. Jean Baubérot : «Ce qu'on nomme communément le "régime concordataire" se met en place. En fait, il est plus exact de le qualifier de système "des cultes reconnus" ", Histoire de la laïcité française, Paris, PUF, 2000, p. 25. Claude Langlois : « Le Concordat a ouvert la voie ; la solution complète s'est trouvée dans le système concordataire qui en résulta, ou si l'on préfère, dans le régime des cultes autorisés ", in " Incertaine actualité du système concordataire en France ", $X X^{e}$ siècle, $n^{\circ}$ 66, avril-juin 2000, p. 107-117, p. 107. Et Émile Poulat : « Avec les Articles organiques, on passe du Concordat au système des cultes reconnus, fondement du régime concordataire qui en est issu et dont ils sont la première pièce ». Notre laïcité publique " La France est une République laïque » (constitution de 1946 et 1958), Paris, Berg International, 2003, p. 72. Nous rejoignons Jean Baubérot sur le fait que « L'expression de régime concordataire indique le poids largement dominant du catholicisme ", Jean Baubérot, Histoire de la laïcité française, op. cit., p. 25.

5. Article X de la Déclaration des droits, dont la formulation contournée porte la trace des débats difficiles qui ont présidé à sa rédaction : « Nul ne doit être inquiété pour ses opinions mêmes religieuses, pourvu que leur manifestation ne trouble pas l'ordre public établi par la loi. » Et Constitution du 3 septembre 1791, Titre $1^{\mathrm{er}}$, « Dispositions fondamentales ».

6. La loi votée dans les tout derniers jours de la Constituante faisait des juifs de France à la fois des Français et des citoyens.

7. Notamment dans le sermon des « Prêtres patriotes », cf. Daniele Menozzi, Les interprétations politiques de Jésus de l'Ancien Régime à la Révolution, Paris, Cerf, 1983, et Rita Hermon-Belot, L'abbé Grégoire, la politique et la vérité, Paris, Seuil 2000, chap. 2 et 3. 8. Pour une discussion sur la nature même du Concordat, Émile Poulat, Notre laicité publique, op. cit., p. 68 et ss.

9. Selon Portalis, parce qu'ils « forment bien moins une religion qu'un peuple », le gouvernement « a cru devoir respecter l'éternité de ce peuple ». Portalis, Discours au Corps législatif, séance du 15 germinal an X, « Sur l'organisation des cultes », Paris, germinal an X, p. 60. Ce qui paraissait très bienveillant mais était en totale contradiction avec le statut de citoyens français dont les avait dotés la loi votée en septembre 1791 par l'Assemblée constituante.

10. Le plus influent d'entre eux : Louis Poujol, Quelques observations concernant les juifs et particulièrement ceux d'Alsace pour fixer l'attention du gouvernement sur la législation des différents peuples à leur égard; sur leurs mours et leurs habitudes, et sur les mesures qu'il pourrait être convenable d'adopter dans la circonstance actuelle, Paris, 1806. 
11. Le décret annulait plus de la moitié de la dette détenue par les prêteurs juifs, soumettait l'exercice de toute activité commerciale à l'obtention d'une patente annuelle, limitait l'immigration juive dans l'Empire et alourdissait les obligations militaires. Une dernière disposition prévoyait l'exemption des « Portugais » du Sud-Ouest, étendue en avril 1810 aux juifs de Paris, du Midi et d'Italie. Ces dispositions valurent à ce décret le surnom de « décret infâme ».

12. Articles organiques du culte catholique, Titre III, LII.

13. Titre VIII, article 260 , « Sur les entraves au libre exercice des cultes ».

14. Connue du pasteur Dupuy, lettre du 18 juin 1803, confirmée par la correspondance de Napoléon, cf. A. Boulay de la Meurthe, Documents sur la négociation du concordat de 1801, et sur les autres rapports de la France avec le Saint-siège en 1800 et 1801, Paris, 1891, T. V, p. 320, n $\circ 2$.

15. Lettre de Portalis, 8 thermidor, an XI, citée par Daniel Robert, Les Églises réformées en France, 1800-1830, Paris, 1961, p. 98.

16. Daniel Robert, op. cit. et Brigitte Basdevant-Gaudemet, Le jeu concordataire dans la France du XIX ${ }^{e}$, Paris, PUF, 1988, p. 236 et ss.

17. L'étude que nous présentons ici a pu être réalisée grâce à l'accueil de la Bibliothèque du Sénat, Paris, VI ${ }^{\mathrm{e}}$.

18. Dans son titre « Droit public des Français », art. 5.

19. Cité par Emmanuel de Waresquiel, Talleyrand, le prince immobile, Paris, Fayard, 2003 (2), p. 636.

20. On rappellera son rôle déterminant dans la rédaction de l'article X de la Déclaration des droits, dans le vote de la loi accordant en janvier 1790 les droits de citoyenneté aux juifs dits « Portugais », mais également dans le vote de la loi de mai 1791, « sur la liberté des cultes », dans la négociation du Concordat puis dans l'adoption du principe et la rédaction des Articles organiques.

21. Pour les autres « corporations » religieuses abolies, le principe suivi avait été celui de la nationalisation des dettes. Sur cette question, Patrick Girard, Les Juifs de France de 1789 à 1860, Paris, Calmann-Lévy, 1976, p. 70 (coll. « Diasporas »).

22. Article VII.

23. À la demande d'un groupe de citoyens juifs qui avaient soutenu la Révolution de Juillet, cf. Patrick Girard, Les Juifs de France de 1789 à 1860, op. cit., p. 88-89. La Charte de 1830 faisait retour à la formule selon laquelle la religion catholique était celle de la grande majorité des Français, mais sans même mentionner la religion du roi.

24. Le 8 janvier 1831, Archives Parlementaires, sous la dir. de M. J. Mavidal et M. E. Laurent, $2^{\mathrm{e}}$ série, T. LXVI, p. 25.

25. Archives Parlementaires, op. cit., T. XXXIII, Paris, 1876, p. 751.

26. Duvergier, J.-B.-M., Collection des lois, décrets, ordonnances, règlements et avis du Conseil d'État, t. 23, 1828 (4), p. 614.

27. M. Dalloz aîné, Jurisprudence générale. Répertoire méthodique et alphabétique de législation, de doctrine et de jurisprudence, t. XIV, Paris, Dalloz, 1853, p. 743.

28. Titre II, article 5.

29. Article VII.

30. À Mayotte, le droit musulman a un statut « quasi officiel », Alain Boyer, Le droit des religions en France, Paris, PUF, 1993, p. 208.

31. Le 8 janvier 1831, Archives Parlementaires, op. cit., T. XXXVI, p. 24-25. Mérilhou associe la forme que ce culte revêt dans le pays même : « le double caractère d'une longue durée dans ses croyances et d'un nombre considérable de sectateurs ». Mais en l'associant à une 
validation plus universelle : celle d'un culte « pratiqué dans presque toutes les régions du monde civilisé ».

32. Ibid., p. 404.

33. On l'a vu supra pour le cas juif, avec les « ordonnances » de 1844. Les textes concernant les protestants datent de 1859.

34. Dalloz, Jurisprudence générale, op. cit., « Culte », T. XIV, p. 758.

35. Séance du 3 ventôse, an III.

36. Décret du 7 vendémiaire an IV sur l'exercice et la police du culte, Titre III, « De la garantie civique exigée des ministres de tous les cultes ».

37. Dalloz, op. cit., p. 764.

38. Cité par R. Anchel, Napoléon et les Juifs, Paris, 1928, p. 63, d'après Pelet de la Lozère, Opinions de Napoléon, Paris, 1833, p. 208.

39. Jean-Michel Leniaud, L'administration des cultes pendant la période concordataire, Paris, NEL, 1988, p. 65. Ou Brigitte Basdevant-Gaudemet, « Le régime des cultes reconnus » in Francis Messner, Pierre-Henri Prélot et Jean-Marie Woehrling, dirs., Traité de droit français des religions, Paris, Litec, 2003, p. 93.

40. Dalloz, op. cit., chap. VI, « Du culte israélite », p. 921.

41. Dichotomie que nous empruntons à René Rémond, « Bonaparte et la paix du Concordat », in « Dieu et la politique », L'Histoire, juillet-août 2004, p. 51.

42. Une circulaire du ministère de l'Intérieur évoque la présence d'une Église consistoriale reconnue par la loi, qu'elle qualifie d'ailleurs aussi bien un peu plus loin d'« établie».

43. 8 janvier 1831, Archives Parlementaires, op. cit., T. XXXVI, p. 404.

44. Ibid., p. 405. Portalis « jeune » avait été l'un des commissaires en charge de l'Assemblée des Notables et du Grand Sanhédrin.

45. Dalloz, op. cit., p. 734.

46. Dalloz, op. cit., « Associations illicites », T. V, 1846, « associations illicites », p. 279-310. Ces autres cultes sont essentiellement l'islam dont l'entrée sur la scène française est saluée à la Chambre dès 1831, mais sans qu'il y soit donné aucune suite et dont la gestion reste confinée à la pratique dérogatoire de la sphère coloniale, et, en métropole, essentiellement des Églises protestantes, voir notamment Sébastien Fath, Une autre manière d'être chrétien en France, Socio-histoire de l'implantation baptiste (1810-1950), Genève, Labor et Fides, 2001.

47. Ibid., p. 297.

48. En Grande-Bretagne, les citoyens de religion juive n'accèdent à la Chambre des Lords qu'en 1859.

\section{RÉSUMÉS}

La très célèbre disposition de l'article 2 de la loi de 1905, la République « ne reconnaît, ne salarie ni ne subventionne aucun culte ", visait une réalité alors familière à tous, celle des cultes dits « reconnus ». Pourtant, non seulement la portée de cette abolition est fréquemment discutée, mais nombre de débats récents ont montré combien la référence aux cultes reconnus est encore 
présente, selon une version de l'histoire qui en attribue sans hésitation la paternité à Bonaparte, dans le cadre de ce que l'on nomme souvent le « système concordataire ». C'est précisément à la genèse de ces cultes reconnus que l'on revient ici, pour en retracer le cheminement progressif à travers les différents régimes politiques de la première moitié du XIX siècle français et pointer le sens très spécifique que revêt le recours à la catégorie de la reconnaissance pour les désigner.

The famous provision of article 2 of the 1905 law according to which the Republic "doesn't recognize, finance or subsidize any religion" referred to the then widely familiar reality of socalled "recognized" religions. However, the meaning of this abolition has been frequently discussed and, many contemporary debates illustrate the extent to which the notion of "recognized religions" is still present, according to a version of history that traces their origin to Bonaparte within the framework of what is often called the concordat. The article deals precisely with the genesis of these recognized religions, in order to trace their status through the different political regimes of the $19^{\text {th }}$ century in France and to illustrate the very specific meaning implied by the use of the category of recognition to refer to them.

La muy célebre disposición del artículo 2 de la ley de 1905, la República "no reconoce, ni paga los salarios, ni subvenciona culto alguno", apuntaba a una realidad entonces conocida por todos, la de los cultos llamados "reconocidos". Sin embargo, no solo el alcance de esta abolición es hoy frecuentemente discutida, sino que algunos debates recientes han mostrado hasta qué punto la referencia a los cultos reconocidos está aún presente, según una versión de la historia que atribuye sin dudarlo la paternidad a Bonaparte, en el marco de lo que se ha dado en llamar "el sistema concordatario". Y precisamente a la génesis de los cultos reconocidos volvemos aquí, para seguir la pista de su progresión a través de los distintos regímenes políticos de la primera mitad del siglo XIX francés, y para señalar el sentido muy específico que reviste el recurso a la categoría del reconocimiento para designarlos.

\section{AUTEUR}

\section{RITA HERMON-BELOT}

Centre d'Études Interdisciplinaires des Faits Religieux 